\title{
Los apoderados, los enemigos: perspectivas de directores escolares en Chile sobre los conflictos escolares
}

\author{
The Families, the Enemies: Chilean Principals' \\ Perspectives on School Conflicts
}

\author{
Felipe Aravena ${ }^{1}$ y Romina Madrid ${ }^{2}$ \\ 'Pontificia Universidad Católica de Valparaíso, Chile. \\ ${ }^{2}$ University of Glasgow, Escocia.
}

\begin{abstract}
Resumen
Ser director es una tarea altamente demandante y estresante, especialmente por la gran cantidad de conflictos que requieren afrontar en el desempeño de ese rol. El objetivo de esta investigación fue identificar los tipos de conflictos que enfrentan los directores con distintos actores de la comunidad, analizando el nivel de intensidad y su frecuencia. Mediante una encuesta en línea enviada a directores de establecimientos municipalizados de Chile ( $\mathrm{n}=395)$ se constata que los conflictos con los adultos son más intensos que con los estudiantes. Dentro de los actores adultos, los apoderados $(41,5 \%)$ son los más conflictivos. Los tipos de conflictos que enfrentan los directores con estos actores se relacionan con denuncias realizadas por estos, la resistencia en acatar y seguir las directrices de la escuela, situaciones de violencia en contra de la escuela, problemas de comunicación, falta de compromiso con la tarea educativa y, finalmente, con la negligencia de la escuela ante situaciones específicas con los estudiantes. Las conclusiones del estudio evidencian la necesidad de desarrollar una comprensión más profunda de los tipos de conflictos y estrategias de resolución empleadas por los líderes escolares.
\end{abstract}

Palabras clave: directores; conflictos; apoderados; familias.

Correspondencia a:

Felipe Aravena

Pontificia Universidad Católica de Valparaíso, Avenida Brasil 2950, Valparaíso, Chile.

Trnava University, Priemyselná 2680/4, 91843 Trnava, Eslovaquia.

felipe.aravena@pucv.cl

(C) 2021 PEL, http://www.pensamientoeducativo.org - http://www.pel.cl

ISSN:0719-0409 DDI:203.262, Santiago, Chile doi: 10.7764/PEL.58.1.2021.5 


\section{Abstract}

Being a school principal is a highly stressful and demanding job, particularly due to the number of conflicts that arise as they perform the role. The purpose of this study was to identify the types of conflicts faced by school principals with different actors and to analyze their intensity and frequency. Through an online survey sent to school principals ( $\mathrm{n}=395)$ working in the municipal sector, it was found that they have much more intense conflicts with other adults than with students. Furthermore, most of the conflicts reported by principals (41.5\%) were with families (parents/guardians). The types of conflicts that principals faced with these actors were organized into the following categories: official complaints against the school, opposition to school rules, violence against the school staff, communication issues, lack of family commitment to educational work, and negligence on the part of the school regarding specific situations with the students. The conclusions highlight the need to develop a deeper understanding of the conflicts faced by school principals.

Keywords: School principals; conflicts; parents; families.

\section{Introducción}

El liderazgo escolar es un factor clave en la mejora de los establecimientos (Brock \& Grady, 2012; Day, Sammons, Hopkins, Leithwood, \& Kington, 2008; Hargreaves \& Fink, 2006). Esto, porque impacta las capacidades, motivaciones y condiciones en las que los profesores desempeñan su labor (Leithwood, Harris, \& Hopkins, 2008). Directores que cuenten con un amplio repertorio de prácticas de liderazgo efectivas tienen más posibilidades de impactar en sus profesores y, en consecuencia, en las oportunidades de aprendizaje de los estudiantes (Leithwood, Louis, Anderson, \& Wahlstrom, 2004; Robinson, Hohepa, \& Lloyd, 2008). A medida que las políticas y reformas educativas enfatizan la importancia del liderazgo, se incrementan las demandas que recaen sobre los directores. Así, una tendencia internacional clara es que el trabajo de los directores se ha vuelto cada vez más demandante (Cowie \& Crawford, 2008; Miller, 2015; Taole, 2013). Esto supone un escenario de alta presión y variadas expectativas por el rol que deben desempeñar los directores en la mejora escolar (Oplatka, 2017).

Desempeñarse como director es una tarea altamente demandante, compleja y estresante (García Garduño, Slater, \& López-Gorosave, 2011; Grubb \& Flessa, 2006) en términos físicos, mentales y emocionales (Steward, 2014). Los problemas que enfrentan los directores en su cargo son diversos, complejos y cambiantes (Montecinos, Bush, \& Aravena, 2018). Pese a existir una apuesta por el liderazgo distribuido, aún pareciera ser que el director es el último responsable de todo lo que sucede en la escuela (Spillane \& Lee, 2014), y la literatura coincide en que las habilidades y disposiciones personales de los directores explican una cantidad significativa del avance o retroceso de la escuela (Leithwood \& Azah, 2014).

Si un área central del trabajo de los directores es desarrollar y cultivar relaciones interpersonales con diversos actores de la comunidad educativa, una habilidad clave que requieren desplegar es la de resolver conflictos de diversa índole con distintos actores educativos (Day, 2005). A pesar de lo extenso de la literatura sobre liderazgo educativo, no sabemos lo suficiente acerca de cuáles son los conflictos que enfrentan los directores en Chile. Esta investigación tiene por objetivo identificar los tipos de conflictos que enfrentan los directores con distintos actores de la comunidad educativa, analizar el nivel de intensidad y su frecuencia. Este artículo se estructura en cinco secciones. Una primera sección presenta una revisión literaria sobre tres temáticas: liderazgo escolar en Chile, demandas del rol directivo y conflictos escolares. En una segunda sección se establece la metodología, para luego dar paso a la presentación de los resultados. En la cuarta sección se comparte la discusión a partir de los resultados, para luego finalizar con las conclusiones del estudio. 


\section{Liderazgo escolar en Chile: políticas e investigación}

La literatura internacional ha confirmado que el liderazgo es la segunda variable que afecta los aprendizajes de los estudiantes (Hitt \& Tucker, 2016). Al parecer, la política pública chilena se ha plegado a esta afirmación, brindando más importancia al liderazgo escolar. Esto ha sido traducido en una serie de políticas sucesivas que han puesto especial énfasis en el liderazgo. Por ejemplo, en 2005 se elaboró el Marco para la Buena Dirección y Liderazgo Escolar (MBDLE); 10 años más tarde, se reeditó una nueva versión estableciendo los lineamientos de un liderazgo efectivo en Chile. El MBDLE (2015) estableció un conjunto de prácticas de liderazgo agrupadas en cinco dimensiones, además de una dimensión de recursos personales desglosados en conocimientos, principios y valores necesarios para impactar positivamente en la comunidad escolar. Asimismo, el Ministerio de Educación (MINEDUC), a través del Centro de Perfeccionamiento, Experimentación e Investigaciones Pedagógicas (CPEIP), ha ofertado cursos de formación profesional para directores, en el marco del Programa de Formación de Directores de Excelencia desde el año 2011 hasta el presente. En 2011, la selección de los directores escolares se trasladó al Servicio Civil a través de un Sistema de Alta Dirección Pública. Este nuevo sistema buscó agregar mayor transparencia y rigurosidad al proceso de selección de directores, comprendiendo que es necesario que los mejores candidatos asuman el cargo. En 2016 se crearon dos Centros de Liderazgo para desarrollar capacidades en líderes escolares, investigar y orientar a la política pública sobre las acciones futuras respecto del liderazgo en los distintos niveles del sistema escolar. Estas iniciativas grafican que la política pública nacional ha comprendido que el liderazgo escolar es un factor clave para la mejora. Asimismo, la producción investigativa sobre liderazgo escolar ha crecido considerablemente durante la última década. Según los resultados del estudio realizado por Aravena y Hallinger (2018), Chile es el país de América Latina con más publicaciones indexadas en revistas internacionales especializadas en liderazgo escolar, concentrando 39\%, solo seguido por México con 22\% del total de la producción. Esto expresa interés por indagar sobre las prácticas de liderazgo que despliegan los directores en Chile, así como sobre las problemáticas que afrontan en el cargo y cómo las políticas influyen en su liderazgo.

\section{El aumento de las demandas y la presión sobre el rol directivo}

Dirigir una escuela es una tarea que conlleva una intensa carga laboral. Se espera que los directores, entre otras tareas, lideren la visión de la comunidad educativa, resuelvan conflictos, administren la escuela y movilicen a los docentes para generar aprendizajes en los estudiantes. Las múltiples y diversas demandas que recaen sobre la escuela se traducen en un gran peso sobre el rol de los directores de escuelas (Friedman, 2002; Oplatka, 2017). De hecho, la carga de trabajo es considerada como uno los aspectos que causan mayor estrés (Fong \& Kleiner, 2004), siendo asociada con la escasez de educadores interesados en asumir como directores (Byrne-Jiménez \& Orr, in Mahfouz, 2018), y con las razones para dejar el cargo (Reames, Kochan, \& Zhu, 2014).

A las demandas tradicionales se suman aquellas provenientes de las políticas de rendición de cuenta y desempeño (Oplatka \& Hemsley-Brown, 2012; Mahfouz, 2018) y las reformas educativas neoliberales (Miller, 2015). Estudios recientes indagan en el potencial efecto de la presión sobre los líderes escolares derivada de las políticas de rendición de cuenta. Por ejemplo, Mitani (2018) estudia el impacto de la legislación norteamericana No Child Left Behind en las condiciones de trabajo, el estrés laboral y la intención de dejar el cargo, concluyendo que los directores experimentan mayor estrés laboral debido a la implementación de la política, especialmente aquellos que no tienen mucha experiencia en el cargo y trabajan en escuelas con una alta población de estudiantes vulnerables.

La literatura relacionada con las demandas del rol directivo se posiciona desde una dimensión subjetiva. Es decir, refiere a los sentimientos y percepciones de los trabajadores de que su trabajo es mucho o muy difícil de desarrollar. Otros autores (Hart \& Staveland, en Oplatka, 2017) señalan que la demanda laboral o carga de trabajo resulta de la interacción entre los requerimientos de la tarea, las circunstancias en las que las tareas se desarrollan, y las habilidades, comportamientos y percepciones del trabajador. 
Los tipos de estresores o determinantes de la alta demanda laboral que experimentan los directores de escuela tienden a ser consistentes en la literatura (Friedman, 2002; Leventis, Papakitsos, Karakiozis, \& Argyriou, 2017; Mahfouz, 2018; Oplatka, 2017). Se distinguen tres tipos de estresores percibidos por los directores: un primer grupo relacionado con el trabajo (por ejemplo, constante cambio, burocracia, incidentes inesperados, etc.); otro, con las relaciones (familias, docentes, etc.), y un tercero con el tiempo (como balance entre familia y trabajo) (Mahfouz, 2018). En el contexto nacional, pareciera ser que un gran estresor del trabajo de los directores son los conflictos escolares (Villalobos, Peña, Aguirre, \& Guerrero, 2017).

\section{Conflictos escolares: desafíos para los directores}

Uno de los principales estresores y demandas del cargo directivo es afrontar los conflictos (Mahfouz, 2018). El conflicto es consustancial a las dinámicas organizacionales (Munduante, Ganaza, \& Alcaide, 1993). Diversos autores han manifestado que las organizaciones, como las escolares, perciben el conflicto como una amenaza a la estabilidad y efectividad, porque se encuentran bajo una lógica de orden y disciplina (Burrell \& Morgan, 1979). No obstante, se ha comprobado que los conflictos pueden generar consecuencias positivas y funcionales, que permiten movilizar a los miembros de la organización (Rahim, 1986) e incluso promover crecimiento y aprendizaje a nivel individual y organizacional (Uline, Tschannen-Moran, \& Perez, 2003).

El conflicto es entendido como un fenómeno atribuido a las diferencias o desacuerdos en actitudes, valores, tareas y políticas entre individuos o grupos, que hacen incompatible el logro de un objetivo (Kantek \& Gezer, 2009; Rahim, 1986; Tjosvold, 2008). Los conflictos tienden a ocurrir con más frecuencia cuando existe una interdependencia individual o altos niveles de confusión entre las partes (Saiti, 2015). La idea no es evitar el conflicto, puesto que es natural, sino gestionarlo de la mejor forma posible (Munduante et al., 1993). Esto es desafiante para los directores, especialmente porque tienden a evitarlo, ya que pocos tienen las competencias para gestionarlo de manera efectiva e implica un alto desgaste energético y emocional (Msila, 2012).

En Chile, la política pública históricamente ha asociado el conflicto a la convivencia escolar. La reciente Política Nacional de Convivencia Escolar (Ministerio de Educación, 2019) refuerza esta idea, estableciendo dentro de la dimensión ética de la convivencia escolar la resolución pacífica y dialogada de los conflictos. En la PNCE se define que los conflictos son situaciones de desacuerdo que se producen en las relaciones cotidianas entre los distintos actores de la comunidad educativa. La PNCE no se hace cargo de una tipología de conflictos, sino que plantea su abordaje de manera genérica. Existe así una perspectiva homogeneizante de los conflictos, sin distinguir entre sus grados de dificultad o intensidad. La tipología de conflictos y sus diversas estrategias de resolución quedan como una deuda pendiente en esta nueva política.

Al igual que la política pública, la investigación en Chile sobre el conflicto ha sido abordada desde una perspectiva de convivencia escolar, disociándola del aprendizaje. Dentro de las escasas investigaciones, un reciente estudio desarrollado por Villalobos y colegas (2017) en 12 liceos públicos reporta que los directores escolares requieren atender una multiplicidad de conflictos, y las estrategias que utilizan para resolverlos también lo son. Cuando los directores enfrentan conflictos con los estudiantes, tienden a ser producidos por su origen cultural o socioeconómico; en cambio, cuando los conflictos son con los adultos, se relacionan con la micropolítica y macropolítica escolar. Contribuir a la literatura nacional con más estudios empíricos que exploren la tipología de conflictos que experimentan los directores escolares es clave para apoyarlos en su resolución. 


\section{Metodología}

La investigación buscó identificar los tipos de conflictos que enfrentan los directores con distintos actores de la comunidad, analizar el nivel de intensidad y su frecuencia. Para ello, se envió una encuesta en línea a todos las y los directores de establecimientos municipalizados pertenecientes a tres regiones del país (Valparaíso, Santiago y Concepción) al finalizar el año escolar 2018. Las tres regiones del país fueron seleccionadas como muestra conveniente (Stake, 2010). La encuesta contenía tres secciones. Una primera sección, para identificar la frecuencia con la que los directores tienen conflictos con diversos actores. Se preguntó ¿Con qué frecuencia ha tenido conflictos con los siguientes actores durante este año? A través de una escala de Likert (nunca, pocas veces, algunas veces, casi siempre y siempre), los participantes indicaron con qué frecuencia tenían conflictos con: sostenedor, otros directores, equipo directivo, docentes, asistentes de la educación, estudiantes y apoderados. Una segunda sección de la encuesta buscó conocer el actor (se utilizaron los mismos actores mencionados en la primera sección) y el tipo de conflicto percibido como más intenso. Se preguntó ¿Con qué actor tuvo el conflicto más intenso?, y se solicitó describir en qué consistió el conflicto. Se entregó la opción de indicar otro actor y se pidió nombrarlo. Finalmente, una tercera sección consideró datos demográficos tales como: nivel de enseñanza que imparte el establecimiento, años de experiencia como director, y género. En una segunda fase cualitativa, se buscó explicar la tendencia de los resultados de la encuesta con un grupo de 13 directores de la $\mathrm{V}$ región. Estos fueron seleccionados por conveniencia (Stake, 2010), puesto que formaban parte de un programa de desarrollo profesional para directores que coordinaban los investigadores de este estudio. Se les solicitó explicar de manera escrita sobre el principal resultado de la encuesta. Es decir, se solicitó explicar por qué los apoderados fueron los actores percibidos como más conflictivos por los directores, siendo invitados a responder cómo podrían explicar esta tendencia. Las respuestas fueron escritas de manera individual por cada uno de los directores. Esto potenció la posibilidad de interpretar las explicaciones proporcionadas de manera detallada, visibilizando ideas y conceptos desplegados en los escritos, que permiten comprender el mundo subjetivo e interpretativo desde el cual se sitúan los participantes (Stake, 2010).

\section{Participantes}

El estudio consideró una muestra por conveniencia no probabilística (Stake, 2010). Esto, porque se consiguieron distintas bases de datos con correos electrónicos de directores de establecimientos municipalizados. La encuesta fue respondida por 422 participantes que se auto reportaron como directores de establecimientos municipalizados. Dentro de esta muestra, se optó por eliminar de la base de datos a directores de escuelas especiales ( $\mathrm{n}=17$ ) y jardines infantiles ( $\mathrm{n}=10$ ), debido a su baja tasa de respuesta. Así, para los efectos de este estudio, los resultados corresponden a un total de 395 directores de establecimientos municipalizados de enseńanza básica y media de tres regiones del país.

La mayoría de los directores se desempeña en escuelas básicas (70,2\%), y del porcentaje restante solo 11,6\% son establecimientos de enseñanza media técnico-profesional. El promedio de la matrícula de los establecimientos es de 341, con una desviación estándar de 298. Un 53,6\% corresponde a establecimientos pequeños, un 24,3\% a establecimientos medianos y un $22,1 \%$, a establecimientos grandes ${ }^{1}$. Un $10,6 \%$ de los establecimientos se encuentra categorizado en alto desempeño por la Agencia de la Calidad en Educación. En tanto, un 8,6\% se encuentra en la categoría de desempeño insuficiente. En cuanto a la experiencia desempeńando el cargo, la mayoría son directores experimentados. Solo 19,2\% seńala tener igual o menos de tres ańos de experiencia, es decir, ser novel (García-Garduño et al. 2011). En cuanto al género, un 54,2\% indica femenino y un 45,8\%, masculino.

1. Para determinar el tamaño de la escuela, se siguió la categorización utilizada por Weinstein y Muñoz (2014), que establece que los establecimientos pequeños poseen menos de 261 estudiantes, los medianos, entre 262 y 470 , y los establecimientos grandes, igual o superior a 471 . 
En tanto, los 13 directores que participaron de la segunda fase del estudio se desempeñan en su mayoría en escuelas básicas pequeñas ( $\mathrm{n}=11$ ), con una matrícula promedio de 247 estudiantes (Min= 91 y Max=1061). Del total de establecimientos, 30,7\% se localiza en áreas rurales y 23,1\% se encuentra en la categoría de desempeño insuficiente. En la muestra no se observan establecimientos de alto desempeńo, escuelas especiales ni jardines infantiles. En relación con los datos sociodemográficos de los directores, ocho son mujeres y cinco, hombres. La mayoría de ellos $(\mathrm{n}=10)$ reporta tener más de cinco años de experiencia como directores y solo tres son noveles, con menos de 2 años de experiencia como director en establecimientos escolares.

\section{Análisis de datos}

Los datos de la encuesta fueron analizados usando estadística descriptiva. Se realizó, además, un análisis de contenido de las respuestas cualitativas de los participantes sobre su descripción del conflicto más intenso. La descripción proporcionada por los directores respecto del tipo de conflicto fue categorizada de manera emergente (Creswell, 2014). Estas respuestas se categorizaron según el actor. Dos investigadores fueron codificando las descripciones hasta llegar a acuerdo (Stake, 2010). Dada la prevalencia del actor apoderados en la categoría intensidad y frecuencia de los conflictos, en este artículo se caracterizan los tipos de conflictos correspondientes únicamente a los asociados a este actor. En la tabla 1 se presenta el esquema de codificación de los tipos de conflictos descritos por los directores, indicando el nombre y definición de la categoría y un respectivo ejemplo. En cuanto a las respuestas de los 13 directores, fueron codificadas de manera emergente e independiente por cada investigador. Una vez realizado este proceso, se procedió a comparar los códigos y, en los casos que no existió coincidencia, se volvió a revisar el código para establecer consensos (Creswell, 2014).

Tabla 1

Esquema de codificación

Categoría

Definición de la categoría

Ejemplo reportado por los directores

Denuncias tanto de las familias hacia la escuela como viceversa. Las primeras incluyen la formalización de acciones legales amparadas en la Superintendencia

Denuncias de la Educación y otros organismos como DAEM y Corporaciones Municipales. Las denuncias de las escuelas a las familias dicen relación con vulneración de derechos de estudiantes.

Denuncia a la Superintendencia por el caso de un estudiante que golpea constantemente a los compañeros de curso $y$ profesionales que trabajan con él. La madre acusa que la escuela no hace nada para ayudar a su hijo.
Conflictos asociados a problemas de comunicación con apoderados que incluyen la aplicación de la normativa

Comunicación o de los procedimientos escolares establecidos. Se hace referencia a la mediación que deben hacer los directores de los conflictos entre docentes y apoderados.
El conflicto con los apoderados es por lo que escriben a través de las redes sociales, que muchas veces es por desinformación.
El conflicto tiene como base la resistencia o reacción de las familias en contra de la escuela. La acción intencionada de las familias con miras a contrarrestar, rechazar, cambiar o ignorar las demandas de la escuela, en particular, sobre problemas o asuntos disciplinarios de sus pupilas/os.
Que un apoderado comprendiera que lo que la escuela hacia (sanciones, actividades formativas, apoyo al estudiante y a ella), era conducente a mejorar la actitud de su hijo y mejorar la convivencia en aula y en patio. Nos veía como enemigos, no como aliados en función de ayudar a su hijo. 
Situaciones de violencia explícita, mayoritariamente verbal, de parte de diferentes actores educativos, los

Violencia que incluyen situaciones de violencia desde las familias hacia directores, docentes u otros miembros de la comunidad escolar, y entre familias.
Apoderada, mujer atrevida, grosera en su trato, no entiende razones, no sabe escuchar, me agrede verbalmente, e intenta golpearme.
Situaciones que se centran en la falta de respuesta de las

Compromiso familias a las demandas de la escuela y la ausencia de un rol formativo.
La falta de compromiso de los padres con el comportamiento de sus hijos, lo que conlleva poco apoyo y comprensión a nuestra labor. Eso genera opiniones encontradas que conllevan a discusiones y roce con ellos.
Situaciones de conflicto generadas por la falta de actuación pertinente del establecimiento, que incluyen

Negligencia el seguimiento e implementación de protocolos o procedimientos de actuación, por ejemplo, en casos de accidentes escolares.
Un estudiante de séptimo año tuvo un golpe en la cabeza y no se le informó al apoderado, debido a que el niño no manifestó molestias durante el día, y posteriormente al llegar a su casa, sintió mareos y fue llevado al hospital por su madre, quedando en observación.

Fuente: Elaboración propia.

\section{Resultados}

Este estudio busca explorar en los tipos de conflictos que enfrentan los directores y la identificación de los actores educativos que aparecen con mayor frecuencia e intensidad en estos conflictos. Los resultados se organizan de acuerdo con lo siguiente: intensidad del conflicto, frecuencia de los conflictos según actor educativo, y tipos de conflictos de directores con apoderados.

\section{Intensidad del conflicto}

En términos de la intensidad del conflicto, la tabla 2 presenta los resultados de la encuesta a directores.

Tabla 2

¿Cuál es el actor con el que ha experimentado el conflicto más intenso durante este año escolar?

\begin{tabular}{ccc} 
Actor & $\mathrm{n}$ & $\%$ \\
\hline Apoderados & 164 & 41,5 \\
\hline Docentes & 74 & 18,7 \\
\hline Estudiantes & 64 & 16,2 \\
\hline Asistentes de la educación & 31 & 7,8 \\
\hline Sostenedor & 23 & 5,8 \\
\hline Equipo directivo & 19 & 4,8 \\
\hline Sin conflicto & 15 & 3,8 \\
\hline Otros & 5 & 1,3 \\
\hline Total & 395 & 100
\end{tabular}

Fuente: Elaboración propia. 
Estos resultados sugieren lo siguiente. Primero, enfrentar conflictos intensos pareciera ser propio y natural del desempeño en el cargo directivo. Esto, porque un escaso número de directores $(\mathrm{n}=15)$ reportó no haber experimentado un conflicto intenso durante el ańo escolar. Segundo, para los directores de este estudio ( $\mathrm{n}=395)$, los conflictos con los adultos son más intensos (83,8\%) que con los estudiantes (16,2\%). Tercero, los directores reportan que dentro de los actores adultos con los que han experimentado los conflictos más intensos es con los apoderados (41,5\%). Considerablemente más abajo siguen los conflictos con los docentes (18,7\%) y los estudiantes (16,2\%). El actor con el que menos se reportan conflictos intensos es con otros (1,3\%). En esta categoría se identifican: gendarmería, concejales, otra directora de la misma comuna, y personas ajenas al establecimiento como, por ejemplo, ladrones o saqueadores. Cuarto, no es posible afirmar que ciertas características de los directores o de la escuela o liceo se asocien a un determinado tipo de conflicto o actor. Esto, porque los análisis estadísticos descriptivos realizados en SPSS indican que no existe una relación estadísticamente significativa entre el actor con el que los directores tienen el conflicto y el tamaño del establecimiento (Chi-square $=7,993$, $\mathrm{p}=, 092)$, los ańos de experiencia en el cargo (Chi-square $=3,901, \mathrm{p}=, 142)$, ni tampoco con el género del director/a (Chi-square $=2,246, \mathrm{p}=, 325)$.

\section{Frecuencia de los conflictos según actor educativo}

En términos de frecuencia de los conflictos que enfrentan los directores según los actores educativos, los datos coinciden con los hallazgos sobre intensidad del conflicto presentados previamente. Los directores reportan tener mayor frecuencia de conflicto (algunas veces y casi siempre) con los apoderados (34,43\%), luego con los estudiantes $(29,87 \%)$, con los docentes $(17,72 \%)$ y asistentes de la educación (16,46\%). Por el contrario, los directores indican nunca tener conflictos con otros directores de establecimientos escolares $(85,57 \%)$, con los sostenedores $(60,51 \%)$, y con su equipo directivo $(58,99 \%)$ (ver tabla 3$)$.

Tabla 3

Frecuencia con que los directores reportan enfrentar conflictos con actores

\begin{tabular}{|c|c|c|c|c|c|c|c|c|c|c|}
\hline \multirow[t]{2}{*}{ Actor } & \multicolumn{2}{|c|}{$\begin{array}{l}\text { Nunca } \\
(0)\end{array}$} & \multicolumn{2}{|c|}{$\begin{array}{l}\text { Pocas veces } \\
\text { (1) }\end{array}$} & \multicolumn{2}{|c|}{$\begin{array}{l}\text { Algunas veces } \\
\text { (2) }\end{array}$} & \multicolumn{2}{|c|}{$\begin{array}{l}\text { Casi siempre } \\
\text { (3) }\end{array}$} & \multicolumn{2}{|c|}{$\begin{array}{l}\text { Siempre } \\
(4)\end{array}$} \\
\hline & $\mathrm{n}$ & $\%$ & $\mathrm{n}$ & $\%$ & $\mathrm{n}$ & $\%$ & $\mathrm{n}$ & $\%$ & $\mathrm{n}$ & $\%$ \\
\hline Sostenedor & 239 & 60,51 & 119 & 30,13 & 26 & 6,58 & 7 & 1,77 & 4 & 0,01 \\
\hline Otro(s) director(es) & 338 & 85,57 & 53 & 13,42 & 4 & 1,01 & 0 & 0 & 0 & 0 \\
\hline Equipo directivo & 233 & 58,99 & 141 & 35,70 & 19 & 4,81 & 2 & 0,5 & 0 & 0 \\
\hline Docentes & 91 & 23,04 & 234 & 59,24 & 66 & 16,71 & 4 & 1,01 & 0 & 0 \\
\hline Asistentes de la educación & 111 & 28,10 & 218 & 55,19 & 60 & 15,19 & 5 & 1,27 & 1 & 0,002 \\
\hline Estudiantes & 93 & 23,54 & 183 & 46,33 & 101 & 25,57 & 17 & 4,3 & 1 & 0,002 \\
\hline Apoderados & 72 & 18,23 & 187 & 47,34 & 118 & 29,87 & 18 & 4,56 & 0 & 0 \\
\hline
\end{tabular}

Fuente: Elaboración propia. 
Estos resultados reportan que la frecuencia de los conflictos percibidos por los directores es mayor con los actores pertenecientes directamente a su comunidad educativa (apoderados, estudiantes, docentes y asistentes de la educación). Esto dado que los actores externos a la comunidad educativa, como sostenedores y otros directores, aparecen con menor frecuencia de conflicto.

\section{Tipos de conflictos de directores con apoderados}

Los datos cuantitativos evidencian que la relación con los apoderados y familias emerge como un nudo crítico en el quehacer directivo, y es el foco de atención más evidente en el ámbito de los conflictos escolares. De manera transversal, los directores describen que los conflictos más intensos y frecuentes ocurren con los apoderados. Dada esta unívoca tendencia, el análisis de los tipos de conflictos más intensos se realizó con foco en los actores apoderados. Estos conflictos fueron tipificados y clasificados en seis categorías, que se observan a continuación (ver tabla 4).

Tabla 4

Tipología de conflictos más intensos con los apoderados según percepción de directores

\begin{tabular}{ccc} 
Categoría & $\mathrm{N}$ & $\%$ \\
\hline Denuncias hacia escuelas/familias & 43 & 26,2 \\
\hline Resistencia & 39 & 23,7 \\
\hline Violencia & 30 & 18,3 \\
\hline Comunicación & 28 & 17,1 \\
\hline Compromiso & 18 & 11 \\
\hline Negligencia & 6 & 3,7 \\
\hline Total & 164 & 100 \\
\hline
\end{tabular}

Fuente: Elaboración propia.

La primera y más extensa categoría, denuncias a escuelas/familias, integra en su mayoría conflictos derivados de acusaciones efectuadas por las familias en contra de las escuelas $(n=33) y$, en una cantidad inferior, conflictos causados por acusaciones hechas por la escuela hacia las familias $(\mathrm{n}=10)$. De las denuncias realizadas por las familias hacia la escuela, la gran mayoría se realizaron ante la Superintendencia de Educación Escolar, organismo que opera desde 2012 en Chile y que tiene por objetivo "fiscalizar, de conformidad a la ley, que los sostenedores de establecimientos educacionales reconocidos oficialmente por el Estado se ajusten a las leyes, reglamentos e instrucciones que dicte la Superintendencia, y fiscalizar la legalidad del uso de los recursos de los establecimientos que reciban aporte estatal.” (Superintendencia de la Educación, s.f).

Las denuncias ante la Superintendencia son particularmente graves para un establecimiento educativo ya que, de constatarse como efectivas, se traducen en consecuencias negativas directas para las escuelas, en términos de multas y sanciones administrativas. Estas consecuencias también inciden en el ámbito individual de los directores, porque usualmente en sus convenios de desempeño se establecen indicadores de logro asociados al número de denuncias por año en la Superintendencia.

Gran parte de las denuncias realizadas por las familias en contra de la escuela tienen que ver con problemas específicos con docentes del establecimiento, por ejemplo: La apoderada hizo demanda en contra de una profesora por agresión física a uno de sus hijos, Denuncia de padre por solicitud de cambio de apoderado, o de sus hijos con otros estudiantes (Reclamos por supuesto Bullying a una alumna, Riña entre alumnas y posterior denuncia a superintendencia y no aceptar errores de sus hijas). 
La segunda categoría de conflictos con apoderados se refiere a experiencias de resistencia a los dictámenes de la escuela. Esta categoría reúne conflictos producidos por la reacción y el no acatamiento de las familias de las normativas, requerimientos o solicitudes de la escuela. También incluye la percepción, de parte de los directores, de que los apoderados no aceptan o no reconocen su responsabilidad o la responsabilidad de su hijo en situaciones de disciplina o conflictos con pares. En su gran mayoría, la resistencia de las familias se asocia a situaciones disciplinarias con los estudiantes. Esta actitud de defensa férrea a los/as hijas/os, por sobre la opinión de la escuela, es la que marca la distancia entre unos y otros. Ejemplos de estos conflictos son Madre no acata compromiso del Manual de Convivencia, Situaciones conductuales en donde los padres no asumen los problemas que tienen sus hijos, el nivel de agresividad que traen consigo producto del clima familiar, Que apoderados comprendan la conducta de sus hijos en la escuela. Como uno de los directores expresa, esta categoría de conflictos pone a las familias como enemigas de la escuela. En sus palabras, "Nos veía como enemigos (los apoderados), no como aliados en función de ayudar a su hijo."

Este tipo de conflictos devela la explícita pugna de poder entre un grupo y otro, y cómo se traduce en un desgaste personal de los directivos. Esta categoría refleja la presión que ejercen las familias en manifestar su abierta oposición a las reglas establecidas por la escuela. Los directivos dejan entrever la percepción de no contar con el respaldo de la credibilidad familiar (tradicionalmente común), en donde los padres respaldan el quehacer de la escuela cuando se refiere al cumplimiento de la disciplina, y sugiere que la alianza ahora existe entre las familias y sus hijos, cuestionando a la escuela. Los actores específicos, docentes o directivos, desaparecen en la descripción de los conflictos y se habla de escuela con un foco más general.

La categoría que sigue, tercera más reportada, es violencia, y describe conflictos con las familias que resultan de insultos verbales y amenazas, e inclusive golpes de estas hacia los directores, y principalmente, hacia los docentes del establecimiento, llegando incluso a estudiantes. Algunos ejemplos son "Agresión verbal de dos apoderados en contra de docentes del establecimiento", "Apoderada falta respeto al director respecto a una situación puntual con su hijo". Resistencia y violencia pueden entenderse como un continuo gradual de situaciones similares que se perciben como de alta intensidad por directores, y reciben más de 37\% del total de los conflictos con los apoderados percibidos como los más intensos. Sin embargo, al describirlo, la violencia adquiere un componente interpersonal, mientras que la resistencia es descrita enfatizando el aspecto institucional.

La categoría comunicación incluye un variado grupo de actividades que aluden a problemas en la comunicación entre escuela y familias. Todos los conflictos en esta categoría expresan la falta de acuerdo en el entendimiento del otro. En estos conflictos, un grupo importante se da entre docentes y apoderados, o entre apoderados, donde los directores intervienen. En esta categoría destaca la percepción de los directores de que las familias están negativamente empoderadas y quieren tener voz y tomar mayores decisiones en el mundo escolar que antes. El conflicto, en esta categoría, es causado por padres que se muestran exigentes, y que abiertamente cuestionan o critican el proceder o los criterios de actuación de las escuelas. Algunos ejemplos son "El conflicto en sí era minimo, pero esto nació por la forma prepotente que se dirigió la apoderada a exigir una solución inmediata", "Hubo una pelea escolar al interior del liceo entre niñas, y sus apoderados, en vez de intentar buscar soluciones, acudieron a la prensa local complejizando más la situación, ya que las niñas fueron expuestas públicamente".

Un 18\% de los conflictos con los apoderados corresponde a la categoría compromiso. Esta categoría dice relación, específicamente, con el compromiso de las familias con la tarea formativa y la falta de cumplimiento sobre acuerdos establecidos. Respecto del primer aspecto, los directores perciben que los conflictos más difíciles con apoderados tienen que ver con la falta de apoyo y responsabilización de estos con la tarea educativa y formativa de sus hijas/os. En ella, se describen prácticas que incluyen desde la inasistencia a actividades escolares hasta la percepción de abandono de los padres de su rol como formadores, y la expectativa de que sea solamente la escuela la que asuma el rol principal en la labor educativa. Por ejemplo, "Un apoderado en particular que insistía 
traspasar su responsabilidad como padres al colegio", "La intransigencia de reconocer su falta de responsabilidad en su rol de padre y apoderado a partir de los compromisos asumidos tanto en el proceso de matrícula como el conocimiento de los reglamentos internos", "Apoderados no apoyan decisiones de los docentes, no aportan con compromisos firmados".

Finalmente, una última categoría de negligencia describe situaciones específicas generadas por la falta de actuación pertinente de la escuela en casos de accidentes escolares y en la aplicación de protocolos de seguridad. Por ejemplo, "Responsabilizar por accidente dentro de la escuela en horario de salida". En esta categoría, los conflictos son producidos por la negligencia de la escuela y los apoderados reaccionan ante esto.

\section{¿Cómo explican los directores estos resultados?}

Una vez recopilados y analizados los datos cuantitativos, se compartieron los resultados con 13 directores de la V región. Ante la pregunta ¿Por qué los conflictos más intensos de los directores se experimentan con apoderados? se encontraron dos grupos de opiniones. Un grupo se centró en los apoderados en sí, aludiendo a las actitudes y a lo que se percibe como un nuevo apoderado, mucho más crítico y demandante de la escuela que antes, y más ausente en sus roles parentales debido al exceso de atención al trabajo, falta de tiempo y despreocupación por la tarea formativa, entre otros. Algunos de estos directores también aluden a padres menos comprometidos con la educación y menos empáticos con la tarea que desarrolla la escuela, los docentes y los directivos. Los directores coinciden en percibir que las demandas y expectativas con las que llegan los apoderados cuando existe una dificultad con la escuela (emocionalmente afectado y buscando soluciones rápidas a sus problemas) dista de lo que, a ellos, en su rol de directores, y la escuela, como organización, pueden dar respuesta. Esto se traduce en frustración por parte de los directores e incrementa la distancia entre unos y otros. En la interpretación de los directores, esto ocurre porque los apoderados no tienen el conocimiento acerca de cómo opera la escuela, no entienden su normativa, tiempos y procedimientos, ni están insertos en la dinámica diaria, por lo que no tienen los elementos para poder analizar la situación de la misma forma en que alguien de la escuela puede hacerlo, y esperan que el director responda rápidamente a sus inquietudes. Un director explicó,

(...) los conflictos con los apoderados son los que ocupan mayor tiempo y generan mayor desgaste en los directivos. A mi juicio, esto se debe a que continuamente los padres exigen la salida de los alumnos que provocan problemas o de profesores con los que tienen dificultades, dando poca consideración al diálogo. Esta situación suele estar acompañada de amenazas a la Superintendencia de Educación, DAEM, alcaldía u opinión pública, lo que constituye una mayor presión y sensación de agobio (Director, cuatro ańos de ejercicio profesional).

De este modo, para este grupo los conflictos con apoderados son resultado del tipo de apoderado, más demandante y crítico del rol de la escuela, al que se ven enfrentados en la actualidad los directores de escuelas municipales.

En cambio, otro grupo de directores centra el análisis en la escuela, y la poca preparación de docentes, asistentes y de los propios directores para vincularse con los apoderados, en especial en situaciones de conflicto. Es una mirada más introspectiva y autocrítica sobre las capacidades de los directores gestionando conflictos con los apoderados. A diferencia del primer grupo, donde se responsabiliza a los apoderados, otros directores expresan una responsabilidad compartida. Respecto de las capacidades profesionales de los docentes y directivos, los directores hablan de la falta de habilidades para contener y comunicarse con los apoderados en situaciones de conflicto, como también para mantener un diálogo permanente con ellos de tal forma que no se llegue a la externalización del conflicto. Los directores también se refieren específicamente al rol directivo en la organización escolar, definiéndolo como el ente que administra las relaciones interpersonales en la escuela y entre la escuela y la comunidad. Un director hace explícito este rol: 
El conflicto no se genera con el director, este es quien tiene que resolver los conflictos que emergen de errores de docentes, asistentes, u otros, por trato, prácticas erróneas, falta de comunicación (...) (Director, 17 años de ejercicio profesional).

De las respuestas de los directores, se interpreta que en la comunidad educativa de los establecimientos municipales existe la expectativa de que el director sea quien resuelva los conflictos interpersonales generados en cualquier nivel de la organización. Esto significa, que las familias asumen que quienes tienen las capacidades de resolver las situaciones conflictivas son ellos, incluso cuando el conflicto es específicamente con un docente u otro actor de la escuela. En parte, serían ellos los que traducen y median entre las demandas de los apoderados y las de la escuela.

Los apoderados esperan que sea el director quien dé respuestas a sus inquietudes, puesto que ellos no tienen toda la información y suponen muchas situaciones que en realidad no ocurren. Debido a esto, su estado emocional se desborda y se enfrenta negativamente con quien lo atiende, quien generalmente es el director (Director, siete años de ejercicio profesional).

\section{Discusión}

En el desempeño del rol directivo, enfrentarse a conflictos es natural e inevitable (Mahfouz, 2018; Rahim, 1986; Saiti, 2015). La fuente de los conflictos se encuentra en la interacción con los distintos actores educativos; esto, porque los desacuerdos y diferencias son propios de las relaciones sociales. Las implicancias de los conflictos en el liderazgo educativo resultan obvias; sin embargo, es un tema que no ha recibido protagonismo, lo que se evidencia en la falta de investigación sobre esta materia.

El objetivo de esta investigación fue identificar los tipos de conflictos que enfrentan los directores con distintos actores de la comunidad, analizar el nivel de intensidad y su frecuencia. Los datos indican que la resolución de conflictos con los adultos es más compleja que con los estudiantes y, en específico, para los directores de este estudio, los apoderados son los adultos con quienes experimentan conflictos con mayor frecuencia e intensidad. La marcada tendencia a identificar a los apoderados como los actores más conflictivos por los directores chilenos, hace explícitas las tensiones existentes entre las escuelas y sus comunidades, como también que los directores parecieran ser quienes lo vivencian cotidianamente mediante múltiples demandas y exigencias. Esto también ha sido reportado por estudios internacionales; por ejemplo, en Estados Unidos, Mahfouz (2018) indicó que los directores, cuando se relacionan con los apoderados, se sienten "listos para una pelea", calificando su relación como altamente compleja, emocionalmente demandante, amenazante o simplemente inútil.

Similarmente, para los directores escolares en Chile, los apoderados son un actor conflictivo (Montecinos, Sisto, \& Ahumada, 2010; Montecinos, Ahumada, Galdames, Campos, \& Leiva, 2015). Desde su perspectiva, los conflictos con los apoderados se producen, en parte, por el tipo de apoderado que se encuentra hoy en el sistema escolar y también por la falta de habilidades y capacidades relacionales y emocionales de la escuela (directivos, docentes, asistentes de la educación) para vincularse con las familias. La descripción de los conflictos intensos con los apoderados sugiere que la relación de la escuela con las familias se encuentra deteriorada, ya que la amenaza y desconfianza mutuas apareció en los relatos de los directores de manera transversal. Estos datos contribuyen a una explicación más profunda sobre lo ya reportado por el estudio de Montecinos et al. (2018), donde se indica que, dentro de la categoría de personas, para un 31\% de los directores es complejo gestionar la relación con las familias y -específicamente- su involucramiento en la escuela.

La marcada prevalencia de conflictos, percibida como frecuente, con apoderados y las propias interpretaciones de los directores de establecimientos municipales sobre los datos permiten afirmar que la tarea de gestionar las relaciones escuela-familia recae de manera individual sobre la figura directiva. Una capacidad necesaria, que 
requiere ser desarrollada por los directores, es gestionar la relación entre la escuela y la familia. Según los datos de este estudio, las familias en Chile -haciendo eco del modelo de mercado existente- tienden a entender su rol como consumidores de educación (López, Aravena, \& Sisto, 2012), y la existencia de la Superintendencia puede incluso haber reforzado este rol. Ante una relación distante con la escuela, lo más efectivo puede ser dirigirse a otro interlocutor, reemplazando a los actores educativos. Cabe preguntarse cómo se apoya a las escuelas en la construcción de un vínculo con las familias y las comunidades en donde aseguremos que sean vistas como aliadas y no enemigas. Avanzar hacia la construcción de espacios para atender las preocupaciones de los apoderados dentro de la escuela y no solo fuera de ella es un paso necesario que no solamente los directores, sino el sistema en su conjunto, requiere dar.

Una de las mayores fuentes de conflicto que experimentan los directores de este estudio con los apoderados se refiere a las denuncias que las familias realizan a la Superintendencia de Educación, las que aluden a la insatisfacción respecto de cómo la escuela da respuesta particularmente a la disciplina entre estudiantes, los problemas con docentes y familias o estudiantes, y los accidentes escolares. En 2012, el año de creación de la Superintendencia, esta recibió más de 4800 denuncias por parte de apoderados; en 2017, aumentaron a más de 12.600. Es decir, en cinco años, la cantidad de denuncias de familias hacia la escuela creció en más de $60 \%$. Es probable que la gran cantidad de conflictos asociados a este tipo de denuncias sea parte de un aprendizaje de las familias de cómo moverse dentro de un sistema que institucionaliza la denuncia. Otros autores ya se han referido a cómo la creación de agencias y políticas de rendición de cuentas transforma la relación entre el Estado y sus ciudadanos y, en particular, la Superintendencia refuerza la idea de que los problemas entre escuelas y familias se resuelven mediante formas de rendición de cuentas marcadas por lógicas de consumo en vez de lógicas democráticas (Montecinos et al., 2015). El aumento de las denuncias y la percepción de intensidad de este tipo de conflictos refuerza la idea de que los directores en Chile han incorporado una subjetividad gerencial propia de la "Nueva Administración Pública”, en donde su quehacer se centra en gestionar amenazas de distintos actores. Todo esto, en un contexto de marketización educativa, en el que los intereses de los padres, como consumidores, presionan a la vez que definen los focos de atención directiva (Montecinos et al., 2010; Montecinos et al., 2015). Tal contexto de mercado no promueve un interés genuino en los directores por promover vínculos de colaboración y alianzas que prevengan conflictos con la comunidad, sino más bien en centrarse en complacer clientes. El rol de la Superintendencia de Educación refuerza institucionalmente una concepción de la relación escuela-familiacomunidad que prioriza las amenazas mutuas y los castigos derivada de estas.

Denunciar implica que la discrepancia entre familias y escuelas no puede resolverse por la acción misma de esta última, sino que es necesario un intermediario externo. Esto sustenta la idea de la baja capacidad de resolución de conflictos de la escuela y, específicamente, del desempeńo directivo, pero también nos habla de un sistema educativo en donde la denuncia se ha instalado como la forma de resolver los conflictos. Cabe mencionar que, con base en la cantidad de denuncias hechas por familias ante la Superintendencia de Educación, se ha ordenado recientemente exigir que, para poder denunciar a la escuela, se constate que la situación ha sido comunicada previamente al establecimiento. Muchos directores se enteran por la misma Superintendencia de Educación de las denuncias realizadas por las familias. Desde este punto de vista, es necesario pensar cómo el diseño del sistema contribuye a entender y abordar los conflictos escuela-familia sacándolos de la escuela, lo que se traduce en restar agencia a los directores para abordar situaciones difíciles con apoderados. En la misma línea, también es un hallazgo identificar que los directores de establecimientos municipales se responsabilizan por atender a una diversidad de situaciones de menor y mayor complejidad en la relación con las familias. Como lo señaló uno de los participantes, "El conflicto no lo genera el director (con las familias), este es el que tiene que resolver el conflicto generado con otros actores (docentes, asistentes de la educación, etc.)”. Lo anterior demuestra una forma de acción individualizada, centrada en la figura del director, quien se sitúa como el primer y último responsable de resolver los conflictos con apoderados. Con esto se refuerza la idea de que el director es el último responsable de todo lo que acontece o no en la escuela (Spillane \& Lee, 2014). 
El desafío para los directores escolares en Chile es cómo visualizar una oportunidad de aprendizaje individual y organizacional gestionando conflictos con los apoderados. Esto, porque de lo contrario, los conflictos pueden ser improductivos, desestabilizar la mejora escolar, convertirse en estresores con alto potencial de desgaste emocional y energético (Mahfouz, 2018; Uline et al. 2003). La consecuencia inmediata es que, en la medida en que los conflictos son más intensos y difíciles de abordar, podría ser que menos candidatos se vean atraídos al cargo directivo y podría aumentar los niveles de abandono del rol. Esta situación, que ya es crítica en otros países, pareciera ser una posible tendencia nacional, especialmente porque se ha reportado que luego de cinco años en el cargo directivo, dos de cada tres directores no tienen ese mismo cargo en la escuela, y la mayor parte de ellos se ha retirado del sistema escolar (40\%) (Valenzuela \& Allende, 2017). Construir un sistema de apoyos e incentivos más efectivos para los directores en Chile pareciera ser clave, especialmente pensando en los altos niveles de abandono y rotativa directiva que ponen en riesgo la estabilidad de los procesos de mejora continua.

Este estudio contribuye a la literatura nacional, identificando y analizando qué tipos de conflictos enfrentan los directores de establecimientos municipales en Chile. Los resultados indican las enormes y complejas demandas a las que deben hacer frente los directores en Chile, y para las cuales no existe una preparación de alta calidad. Asimismo, ilustran cómo los conflictos más intensos de los directores, así como la gestión de las relaciones con las familias, responden a tensiones que escapan a la formación directiva y son propias de las características del sistema educativo. Estos hallazgos no están aislados de lo que implica liderar en un contexto de mercado y competición, en donde los directores son responsabilizados por resultados y matrículas (Carrasco \& Fromm, 2016; Weinstein et al., 2012). Los resultados expuestos pretenden contribuir a los programas de formación en liderazgo en temáticas de resolución de conflictos, especialmente porque existe una necesidad no solo de formar en una comprensión más profunda sobre la tipología y estrategias de resolución de conflictos, sino también en cómo poder construir espacios de colaboración y relaciones de confianza mutua entre los directores y las familias. En la medida en que los directores en Chile reconfiguren a los apoderados como aliados estratégicos de la mejora escolar y dejen de ser vistos como enemigos, las posibilidades reales de que las escuelas mejoren creando más y mejores oportunidades de aprendizaje para los estudiantes pueden ser mayores.

El artículo original fue recibido el 28 de junio de 2019

El artículo revisado fue recibido el 5 de mayo de 2020

El artículo fue aceptado el 3 de junio de 2020

\section{Referencias}

Aravena, F. \& Hallinger, P. (2018). Systematic review of research on educational leadership and management in Latin America, 1991-2017. Educational Management Administration \& Leadership, 46(2), 207-225. https://doi.org/10.1177/1741143217745882

Brock, B. \& Grady, M. (2012). The daily practices of successful principals. California, CA: SAGE.

Burrell, G. \& Morgan, G. (1979). Sociological paradigms and organizational analysis. Londres, Reino Unido: Heinemann.

Carrasco, A. \& Fromm, G. (2016). How local market pressures shape leadership practices: evidence from Chile. Journal of Educational Administration and History, 48(4), 290-308. https://doi.org/10.1080/00220620.2016.1210584

Creswell, J. (2014). Research design: Qualitative, Quantitative and Mixed Methods Approaches (4ta edición). Thousand Oaks, CA: Sage Publications.

Cowie, M. \& Crawford, M. (2008). "Being" a novice principal in Scotland. Journal of Educational Administration, 46(6), 676-689. https://doi.org/10.1108/09578230810908271 
Day, C. (2005). Sustaining success in challenging contexts: Leadership in English schools. Journal of Educational Administration, 43(6), 573-583. https://doi.org/10.1108/09578230510625674

Day, C., Sammons, P., Hopkins, D., Leithwood, K., \& Kington, A. (2008). Research into the impact of school leadership on pupil outcomes: Policy and research contexts. School Leadership and Management, 28(1), 5-25. https://doi.org/10.1080/13632430701800045

Fong, K. \& Kleiner, B. H. (2004). New development concerning the effect of work overload on employees. Management Research News, 27(4/5), 9-16. https://doi.org/10.1108/01409170410784419

Friedman, I. A. (2002). Burnout in school principals: Role related antecedents. Social Psychology of Education, 5(3), 229-251. https://doi.org/10.1023/A:1016321210858

García Garduño, J. M., Slater, C., \& López-Gorosave, G. (2011). Beginning elementary principals around the world. Management in Education, 25(3), 100-105. https://doi.org/10.1177/0892020611403806

Grubb, W.N. \& Flessa, J. (2006). A job too big for one: Multiple principals and other nontraditional approaches to school leadership. Educational Administration Quarterly, 42, 518-550. https://doi.org/10.1177/0013161X06290641

Hargreaves, A. \& Fink, D. (2006). Sustainable leadership. San Francisco, CA: Jossey-Bass Education Series.

Hitt, D. \& Tucker, P. (2016). Systematic Review of Key Leader Practices Found to Influence Student Achievement: A Unified Framework. Review of Educational Research, 86(2), 531-569. https://doi.org/10.3102/0034654315614911

Kantek, F. \& Gezer, N. (2009). Conflict in schools: Student nurses' conflict management styles. Nurse Education Today 29(1), 100-107. https://doi.org/10.1016/j.nedt.2008.07.007

Leithwood, K. \& Azah, V. (2014). Elementary principals' and vice-principals' workload study: Final report. Unpublished manuscript.

Leithwood, K., Louis, K. S., Anderson, S., \& Wahlstrom, K. (2004). How leadership influences student learning. Nueva York, NY: Wallace Foundation.

Leithwood, K., Harris, A., \& Hopkins, D. (2008). Seven strong claims about successful school leadership. School leadership and management, 28(1), 27-42. https://doi.org/10.1080/13632430701800060

Leventis, C., Papakitsos, E. C., Karakiozis, K., \& Argyriou, A. (2017). Work-related stress and burnout factors of principals in regional Greece: A historical perspective. Journal of Research Initiatives, 3(1), 1. Recuperado de https://digitalcommons.uncfsu.edu/jri/vol3/iss1/1/

López, V., Aravena, F., \& Sisto, V. (2012). "Red Light" in Chile: Parents Participating as Consumers of Education Under Global Neoliberal Policies. En H. Cuadra-Montiel (Ed.), Globalization-Education and Management Agendas (pp.28-54). Rijeka, Croacia: IntechOpen. https://doi.org/10.5772/50305

Mahfouz, J. (2018). Principals and stress: Few coping strategies for abundant stressors. Educational Management Administration \& Leadership, 48(3), 440-458. https:/doi.org/10.1177/1741143218817562

Miller, P. (2015). Leading remotely: exploring the experiences of principals in rural and remote school communities in Jamaica. International Journal of Whole Schooling, 11(1), 35-53. Recuperado de http://www.wholeschooling. net/Journal_of_Whole_Schooling/IJWSIndex.html

Ministerio de Educación. (2019). Política Nacional de Convivencia Escolar (PNCE). Recuperado de http://convivenciaescolar.mineduc.cl/politica-nacional/

Mitani, H. (2018). Principals' working conditions, job stress, and turnover behaviors under NCLB accountability pressure. Educational Administration Quarterly, 54(5), 822-862. https://doi.org/10.1177/0013161X18785874

Montecinos, C., Bush, T., \& Aravena, F. (2018). Moving the school forward: Problems reported by novice and experienced principals during a succession process in Chile. International Journal of Educational Development, 62, 201-208. https://doi.org/10.1016/j.ijedudev.2018.04.004

Montecinos, C., Ahumada, L., Galdames, S., Campos, F., \& Leiva, M. V. (2015). Targets, threats and (dis) trust: The managerial troika for public school principals in Chile. Education policy analysis archives, 23, 87. https://doi.org/10.14507/epaa.v23.2083

Montecinos, C., Sisto, V., \& Ahumada, L. (2010). The construction of parents and teachers as agents for the improvement of municipal schools in Chile. Comparative Education, 46(4), 487-508. https://doi.org/10.1080/03050068.2010.519481

Msila, V. (2012). Conflict Management and School Leadership. Journal of Communication, 3(1), 25-34. https://doi.org/10.1080/0976691X.2012.11884792 
Munduante, L., Ganaza, J., y Alcaide, M. (1993). Estilos de gestión del conflicto interpersonal en las organizaciones. Revista de Psicología Social, 8(1), 47-68. https://doi.org/10.1080/02134748.1993.10821669

Oplatka, I. (2017). Principal workload: Components, determinants and coping strategies in an era of standardization and accountability. Journal of Educational Administration, 55(5), 552-568. https://doi.org/10.1108/JEA-06-2016-0071

Oplatka, I. \& Hemsley-Brown, J. (2012). The research on school marketing: current issues and future directions-an updated version. En I. Oplatka \& J. Hemsley-Brown (Eds.), The management and leadership of educational marketing: Research, practice and applications (pp. 3-35). Emerald Group Publishing Limited.

Rahim, M. (1986). Managing conflicts in organizations. Nueva York, NY: Praeger.

Reames, E. H., Kochan, F. K., \& Zhu, L. (2014). Factors influencing principals' retirement decisions: A southern US perspective. Educational Management Administration \& Leadership, 42(1), 40-60. https://doi.org/10.1177/1741143213499254

Robinson, V., Hohepa, M., \& Lloyd, C. (2008). School leadership and student outcomes: identifying what works and why: Best evidence synthesis iteration [BES]. Wellington, Nueva Zelanda: New Zealand Ministry of Education.

Saiti, A. (2015). Conflicts in schools, conflict management styles and the role of school leader: A study of Greek primary school educators. Educational Management Administration \& Leadership, 43(4), 582-609. https://doi.org/10.1177/1741143214523007

Spillane, J. \& Lee, L. (2014). Noviceschool principals' sense of ultimate responsibility: Problems of practice in transitioning to the principal'soffice.EducationalAdministration Quarterly,50(3),431-465.https://doi.org/10.1177/0013161X13505290

Stake, R. E. (2010). Qualitative research: Studying how things work. Nueva York, NY: The Guildford Press.

Steward, J. (2014). Sustaining emotional resilience for school leadership. School Leadership \& Management, 34(1), 52-68. https://doi.org/10.1080/13632434.2013.849686

Superintendencia de la Educación. (s.f). Conoce la Superintendencia de la Educación. Recuperado de https://www.supereduc.cl/la-institucion/que-es-la-superintendencia-de-educacion/

Taole, M. (2013). Exploring principals' role in providing instructional leadership in rural high schools in South Africa. Studies in Tribes and Tribals, 11(1), 75-82. https://doi.org/10.1080/0972639X.2013.11886668

Tjosvold, D. (2008). The conflict positive organization: It depends upon us. Journal of Organizational Behavior, 29(1), 19-28. https://doi.org/10.1002/job.47

Uline, C. L., Tschannen-Moran, M., \& Perez, L. (2003). Constructive conflict: How controversy can contribute to school improvement. Teachers College Record, 105(5), 782-815. Recuperado de https://www.tcrecord.org/Content.asp?ContentId=11137

Valenzuela, J.P. y Allende, C. (2017). Trayectoria de los directores chilenos en la última década: Primeros antecedentes para politicas públicas (archivo PDF). Recuperado de https://www.lidereseducativos.cl/wp-content/ uploads/2017/12/Valenzuela-Trayectoria-de-los-directores-chilenos-en-la-u\%CC\%81ltima-de\%CC\%81cadaPrimeros-antecedentes-para-poli\%CC\%81ticas-pu\%CC\%81blicas.pdf

Villalobos, C., Peńa, J., Aguirre, E., y Guerrero, M. (2017). Liderazgo escolar y conflictos socioeducativos. Un estudio exploratorio en liceos públicos chilenos. Revista Calidad en la Educación, (47), 81-111. https://doi.org/10.4067/S0718-45652017000200081

Weinstein, J. \& Muñoz, G. (2014). When duties are not enough: principal leadership and public or private school management in Chile.SchoolEffectivenessandSchoolImprovement,25(4),651-670.https://doi.org/10.1080/09243453.2013.792850

Weinstein, J., Muńoz, G., \& Marfán, J. (2012). Liderar bajo presión: las estrategias gestionadas por los directores de escuela para alcanzar los resultados comprometidos. En J. Weinstein \& G. Muñoz (Coords.), ¿Qué sabemos sobre los directores de escuela en Chile? (pp. 219-254). Santiago, Chile: Fundación Chile. 